
https://doi.org/10.1007/s00103-020-03169-w Online publiziert: 5 . Juni 2020

(c) Der/die Autor(en) 2020

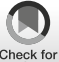

Bundesgesundheitsbl 2020 63:872-880

Michaela Veronika Bonfert ${ }^{1}$ Corinna Börner' ${ }^{1}$ Lucia Gerstl' • Iris Hannibal' Nina Mathonia ${ }^{1}$. Kristina Huß ${ }^{1}$ Birte Rahmsdorf' ${ }^{1}$ Christina Kainz' ${ }^{1}$ Birgit Klose ${ }^{1}$. Helene Koenig' - Giada Urban' ${ }^{\prime}$ Paul Schandelmaier' ${ }^{1}$ Tabea Renner' . Lucia Albers ${ }^{2,3} \cdot$ Sandro Manuel Krieg ${ }^{3} \cdot$ Nico Sollmann ${ }^{4} \cdot$ Florian Heinen ${ }^{1}$ Mirjam Natascha Landgraf'

' Abteilung für Pädiatrische Neurologie, Entwicklungsneurologie, Sozialpädiatrie und LMU Zentrum für Entwicklung und komplex chronisch kranke Kinder - iSPZ Hauner, Dr. von Haunersches Kinderspital, LMU Klinikum, München, Deutschland

${ }^{2}$ Institut für Soziale Pädiatrie und Jugendmedizin, Ludwig-Maximilians-Universität, München, Deutschland

${ }^{3}$ Neurochirurgische Klinik und Poliklinik, Technische Universität, Klinikum rechts der Isar, München, Deutschland

${ }^{4}$ Abteilung für Diagnostische und Interventionelle Neuroradiologie, Technische Universität, Klinikum rechts der Isar, München, Deutschland

\section{Migräne im Kindes- und Jugendalter - Ausblick auf innovative Behandlungsansätze im Rahmen multimodaler Therapiekonzepte}

\section{Einleitung}

Die Migräne zählt mit mehr als einer Milliarde Betroffenen zu den weitverbreiteten und zunehmend als besonders wichtig erkannten Gesundheitsproblemen weltweit [1-3]. In Europa betrifft die Migräne ca. 50 Mio. Menschen [4, 5]. Mit Beginn der Erkrankung und ihren Vorläufern im Kleinkindesalter sowie ihrer Prävalenz in der Pubertät ist die Migräne eine typische Erkrankung des Jugendlichen [6-8]. Als primäre Kopfschmerzerkrankung ist sie trotz hoher Verbreitung und hoher klinischer Relevanz in Verständnis, Forschung und Förderung beispiellos unterschätzt und unterrepräsentiert $[2,9,10]$. Dabei führt sie häufig zu jahrelang andauernden Einschränkungen im Lebensalltag der betroffenen jungen PatientInnen und geht mit einem relevanten Chronifizierungsrisiko einher [11-13].

Nach Diagnosesicherung gilt insbesondere für Patienten mit einer hohen Krankheitslast und somatoformen und/ oder psychiatrischen Komorbiditäten die
Behandlung im Rahmen eines patientenzentrierten, multimodalen Therapiekonzepts im Sinne des biopsychosozialen Modells als Goldstandard, ein Setting, welches hohe spezifische Erfahrung, stabile Ressourcen und geeignete klinischambulante und klinische Strukturen voraussetzt [14].

Trotz der Vielfalt an pharmakologischen Therapieoptionen, sowohl zur akuten als auch zur prophylaktischen Behandlung, besteht die dringliche Notwendigkeit, innovative und effektive nichtpharmakologische Migränetherapien $\mathrm{zu}$ entwickeln $[15,16]$. Dies gilt umso mehr für die Gruppe der Kinder und Jugendlichen, als hier die RisikoNutzen-Relation für die Pharmakoprophylaxe besonders ins Gewicht fällt [15]. Durch Fortschritte im Verständnis der Migränepathophysiologie rücken Methoden der therapeutischen Neuromodulation zunehmend in den Fokus der neurologischen Forschung [17, 18]. In diesem Zusammenhang bietet das Konzept der repetitiven peripheren $\mathrm{Ma}$ gnetstimulation im Bereich der Schulter-
Nacken-Muskulatur einen aussichtsreichen und piloterprobten Ansatzpunkt, da die Assoziation von Muskelschmerzen und/oder Verspannungen in diesem Bereich zur Migräne, insbesondere für das Kindes- und Jugendalter, hinreichend belegt ist $[19,20]$.

In dieser Übersichtsarbeit stellen wir dar, welche Schwerpunkte die biopsychosoziale Betreuung von Kindern und Jugendlichen mit Migräne ausmachen. Vor diesem Hintergrund sowie anhand der Diskussion bezüglich der aktuellen Forschung zur Migränepathophysiologie werden die verschiedenen innovativen neuromodulatorischen Therapieansätze vorgestellt, eingeordnet und dabei der zukünftige Stellenwert der repetitiven peripheren Magnetstimulation skizziert.

\section{Epidemiologie}

Kopfschmerzen sind insgesamt ein sehr häufiges Symptom im Kindes- und Jugendalter, die 1-Monats-Prävalenz beträgt bei Jugendlichen bis $80 \%$. Eine relevante Zahl leidet dabei an einer 
primären Kopfschmerzerkrankung allen voran den Spannungskopfschmerzen und der Migräne [6, 11, 21]. Im pädiatrischen Kollektiv liegt die Prävalenz der gesicherten Migräne bei bis zu 10-20\%, der wahrscheinlichen Migräne bei $15 \%$ und der chronischen Migräne bei $0,1 \%$. Die Prävalenz der Migräne nimmt im Verlauf der Adoleszenz stetig $\mathrm{zu}[7,8,11,22]$. Eine Remission in der Pubertät ist bei $30-60 \%$ der Patienten zu erwarten, wobei es bei ca. einem Drittel dieser Patienten zu einem erneuten Auftreten der Symptomatik in höherem Lebensalter kommt. Ein Beginn der Erkrankung im jungen Alter ist mit einer späteren Persistenz der Beschwerden korreliert [12, 23-25]. Das Progressionsrisiko von episodischer $\mathrm{zu}$ chronischer Migräne (Anstieg der Kopfschmerztage auf $>15$ pro Monat, davon mind. 8 Migränetage, Persistenz der hohen Frequenz über $>3$ Monate) liegt im Erwachsenenalter bei ca. 3\% [26]. Hier gelten eine hohe Kopfschmerzfrequenz, der Medikationsübergebrauch und eine gesteigerte Schmerzempfindlichkeit der Haut (kutane Allodynie) als Risikofaktoren für eine Progression von episodischer $\mathrm{zu}$ chronischer Migräne. Mit steigender Anzahl insbesondere an psychiatrischen Komorbiditäten (Depression, Angst) und Komorbiditäten aus dem Formenkreis der Schmerzsyndrome steigt das Risiko ebenfalls [27]. Eine Metaanalyse zeigte im Kindes- und Jugendalter ähnliche Risikofaktoren auf [26].

\section{Bedeutung der Migräne für die Betroffenen}

Obwohl die Migräne eine solch hohe Prävalenz aufweist, ist sie nach wie vor unterdiagnostiziert und oft unzureichend behandelt $[5,10,28]$. Die Kopfschmerzforschung gilt zudem als ein bei Weitem unterfinanziertes Gebiet [9]. Dies steht im krassen Gegensatz zur hohen Bedeutung der Erkrankung für die einzelnen Betroffenen und für die Gesellschaft durch ihre periodischen „Knock-out-Attacken“. Die Patienten erleben eine besondere Beeinträchtigung ihrer Lebensqualität, bedingt durch das Schmerzerleben, aber auch die direkten und indirekten somatischen, psychischen und sozialen Folgen, die über die akute Beeinträchtigung während einer Attacke weit hinausgehen [29-31]. Die Migräne führt zu einer volkswirtschaftlich relevanten Einschränkung der Produktivität des Einzelnen (im Durchschnitt 10 Arbeitstage pro Jahr) und der Gesellschaft [2, 31-33]. Die im europäischen Gesundheitssystem entstehenden jährlichen direkten medizinischen Kosten betragen pro Patient mit episodischer Migräne $746 €$, bei chronischer Migräne das Dreifache. Insgesamt sind für Europa direkte und indirekte jährliche Kosten von $€ 18,5 \mathrm{Mrd}$. errechnet [34]. Die volkswirtschaftlichen Kosten der kindlichen Migräne selbst sind bisher nicht eingehend untersucht, obwohl aufgrund von Krankheitstagen eines Kindes auch von häufigen Fehltagen der Eltern ausgegangen werden muss [31]. Die Lebensqualität erfährt bei den betroffenen Kindern und Jugendlichen eine Beeinträchtigung, aber eben auch bei den Eltern [13, 22].

\section{Klinisches Bild der Migräne}

Die Migräne ist gekennzeichnet durch wiederholte akute Kopfschmerzepisoden von mittlerer bis hoher Intensität (in der Regel $>6$ von 10 auf der numerischen Analogskala von 0 bis 10). Für die Migräne im Kindes- und Jugendalter können folgende klinischen Besonderheiten genannt werden: meist Migräne ohne Aura (60-80\%); kürzere Dauer der einzelnen Attacken (2-72 h, wobei auch Attackendauern mit $<2 \mathrm{~h}$ berichtet werden), häufiger bilaterale als unilaterale, in der Regel frontale Lokalisation. Häufig besteht eine vegetative Symptomatik in Form von Übelkeit, Erbrechen (wird dann oft als erleichternd empfunden) oder „nur“ abdominellem Schmerz. Die kindliche Migräne beginnt oft in den frühen Morgenstunden, führt $\mathrm{zu}$ einer Unterbrechung von eigentlich gerne durchgeführten Beschäftigungen und $\mathrm{zu}$ einem Rückzug mit „Abschottung" und strikter Vermeidung (auch geringer, Beispiel „Treppensteigen“) körperlicher Aktivität. Oft wird ein eindrucksvoll „imperativer“ Schlafdrang berichtet, nach dem Schlaf präsentieren sich die Kinder dann meist „wieder gesund“. Vorläufersymptome (Premonitory Symptoms) i. S. von autonomen, psychischen oder neurologischen Symptomen (Stimmungsschwankungen, Konzentrationsstörungen, Inappetenz, Heißhungerattacken, Müdigkeit, auffallende Blässe, Lichtempfindlichkeit, Nackenschmerzen, Nackensteife, häufiger Harndrang) werden häufig beobachtet $[8,35]$. Es ist also herauszustellen, dass die Migränesymptomatik im Sinne des Konzepts „migraine is a brain state“ viel mehr ist als nur das Symptom „Schmerz“. Konkret beschreibt sie ein ganzes Spektrum zentraler, peripherer und autonomer Symptome mit den für die Kinder- und Jugendmedizin wichtigen Charakteristika des jeweiligen Entwicklungsalters [9, 36].

\section{Diagnosestellung und Differenzialdiagnostik}

Eine Migräne wird auf Grundlage einer sorgfältigen Anamneseerhebung und internistischer und (kinder-)neurologischer Untersuchung primär klinisch diagnostiziert. Es finden die Kriterien der Internationalen Klassifikation von Kopfschmerzerkrankungen (ICHD 3) Anwendung [37]. In der Regel ist bei stimmigem Gesamtbild mit Beschwerdefreiheit zwischen den einzelnen Episoden keine weitere Diagnostik erforderlich. Eine positive Familienanamnese kann auf eine Migräne hinweisen [35]. Eine weiterführende diagnostische Abklärung ist insbesondere bei atypischen Symptomen oder Verläufen (z.B. plötzliche Zunahme der Kopfschmerintensität oder -frequenz) und bei klinischen Symptomen, die auf eine (weitere) neurologische Erkrankung (z.B. Gefäßsymptomatik mit transitorischer ischämischer Attacke, Schlaganfall, Ursachen einer intrakraniellen Druckerhöhung) hinweisen, indiziert [38].

\section{Multimodale Betreuung}

Für eine so komplexe, wie beeinträchtigende neurologische Erkrankung wie die Migräne gilt die Behandlung im Rahmen eines multimodalen Therapiekonzeptes als Goldstandard, insbesondere dann, 
wenn sie mit einer hohen Krankheitslast einhergeht, sich eine Chronifizierung abzeichnet oder Komorbiditäten aus dem somatoformen oder psychiatrischen Formenkreis vorliegen [14]. Übergeordnete Ziele der Betreuung von Kindern und Jugendlichen mit Migräne sind die Verringerung bis Vermeidung einer Einschränkung des Alltags und der Lebensqualität durch die Erkrankung. Daneben gilt es einer Chronifizierung der Symptomatik i. S. einer Zunahme der Kopfschmerzhäufigkeit und einem Übergang in eine funktionelle (somatoforme/psychogene) Störung/somatische Belastungsstörung vorzubeugen. Kurzbis mittelfristige Ziele sind in der Regel: Reduktion der Kopfschmerzfrequenz, Reduktion der Dauer der einzelnen Kopfschmerzepisode, Verbesserung des Umgangs mit Schmerzen, Vermeidung von Vermeidungsstrategien (z.B. Fernbleiben von der Schule), Vermeidung eines Analgetikaabusus [15].

In der Regel wird nach Diagnosesicherung ein patientenzentriertes Therapiekonzept definiert, das je nach Verlauf, individuellem Bedarf und Ressourcen gemeinsam mit dem Patienten erweitert oder reduziert werden kann. Werden die Möglichkeiten der Grundversorgung überschritten, ist es sinnvoll, eine Betreuung in einem interdisziplinären, „dreidimensionalen“ Team aus Pädiatrie, Physiotherapie und Psychologie in einem spezialisierten Zentrum mit der Familie zu diskutieren und anzustreben [14]. Die Betreuung dieser Patienten ist zeitaufwendig und erfordert die Einbettung in eine klar definierte Struktur. Die konkreten Vorstellungsintervalle werden dabei individuell flexibilisiert, die Möglichkeit zu Telefonkontakten im Intervall kann die Adhärenz fördern. Der hohe Stellenwert der Adhärenz/Compliance und der Selbstkonzeptualisierung und Selbstverantwortlichkeit des Patienten für den Erfolg der Behandlung des eigenen Kopfschmerzes wird im Rahmen des multimodalen Konzepts betont und bleibt eine zentrale Herausforderung an einen gelingenden Versorgungspfad [39].

Bundesgesundheitsbl 2020 63:872-880 https://doi.org/10.1007/s00103-020-03169-w (c) Der/die Autor(en) 2020

M. V. Bonfert · C. Börner · L. Gerstl · I. Hannibal · N. Mathonia · K. Huß · B. Rahmsdorf · C. Kainz · B. Klose · H. Koenig · G. Urban · P. Schandelmaier · T. Renner · L. Albers · S. M. Krieg · N. Sollmann · F. Heinen - M. N. Landgraf

\section{Migräne im Kindes- und Jugendalter - Ausblick auf innovative Behandlungsansätze im Rahmen multimodaler Therapiekonzepte}

\section{Zusammenfassung}

Die Migräne ist auch im Kindes- und Jugendalter ein häufiges, aber in klinischer Versorgung und Wissenschaft oft unterrepräsentiertes Krankheitsbild. Gerade im Kindes- und Jugendalter bestehen relevante Einschränkungen der Lebensqualität durch das (häufige) Schmerzerfahren. Bedingt durch die entwicklungsspezifisch hohe Vulnerabilität des adoleszenten Gehirns besteht ein hohes Chronifizierungs- und Persistenzrisiko bis ins Erwachsenenalter hinein. In diesem Beitrag werden die Bestandteile eines patientenzentrierten, multimodalen Therapiekonzepts dargestellt. Darüber hinaus werden die aktuellsten Erkenntnisse zu den pathophysiologischen Grundlagen der Migräneerkrankung beleuchtet, nach denen
Migräne durch einen sich phasenweise verändernden Funktionszustand des Gehirns entsteht (Stichwort: „migraine is a brain state“). Auch periphere Komponenten wie Muskelschmerzen, -verspannungen und -triggerpunkte spielen eine wichtige Rolle. Vor diesem Hintergrund werden nichtpharmakologische innovative Therapieansätze vorgestellt, die auf dem Prinzip der Neuromodulation beruhen, mit Fokus auf der repetitiven peripheren Magnetstimulation.

Schlüsselwörter

Repetitive periphere Magnetstimulation . Trigemino-cervikaler Komplex · Myofasziale Triggerpunkte $\cdot$ Neurostimulation . Magnetstimulation

\section{Migraine in childhood and adolescence-neurostimulation as a future innovative approach in terms of a multimodal treatment regimen}

\begin{abstract}
Although migraine is a relevant health issue in children and adolescents, clinical care and research are still underrepresented and underfunded in this field. Quality of life can be significantly reduced when living with frequent episodes of pain. Due to the high level of vulnerability of the developing brain during adolescence, the risk of chronification and persistence into adulthood is high. In this narrative review, we describe the corner stones of a patient-centered, multimodular treatment regimen. Further, an update on the pathophysiology of migraine is given considering the concept of a periodically oscillating functional state of the brain in
\end{abstract}

migraine patients ("migraine is a brain state"). Besides central mechanisms, muscular structures with the symptoms of muscular pain, tenderness, or myofascial trigger points play an important role. Against this background, the currently available nonpharmacological and innovative neuromodulating approaches are presented focusing on the method of repetitive peripheral magnetic stimulation.

\section{Keywords}

Repetitive peripheral magnetic stimulation . Trigemino cervical complex - Myofascial triggerpoints · Neurostimulation · Magnetic stimulation

\section{Diagnosegespräch und Psychoedukation}

Ziel des ärztlichen Diagnosegesprächs ist es, dem Patienten eine Konzeptualisierung des ihm eigenen Kopfschmerzes auf dem Boden des biopsychosozialen Modells zu ermöglichen. Durch Darstellung der Diagnose und ihrer biologischen Grundlagen lassen sich Fehlattributionen realisieren, reduzieren und eliminieren
[35]. Es werden auf evidenzbasierter Grundlage Lebensstilfaktoren und individuelle körperliche und psychische Auslösefaktoren diskutiert: Schlafmangel oder unregelmäßiger Schlaf, körperliche Inaktivität, Übergewicht, Alkohol(>2 Getränke/Woche), Koffein- (>2 Tassen Kaffee/Tag oder deren Äquivalent in Cola oder Energydrinks) und Nikotinkonsum, geringe Flüssigkeitszufuhr (kontrovers), Freizeitstress (zu wenig 
regenerative, nicht verplante Zeit), Leistungsdruck (schulischer Stress, Erwartungsdruck der Eltern), sozialer Druck (Peergroup, Mobbing), Schule (als ungerecht empfundene Behandlung durch Lehrer) und familiäre Konflikte [40, 41]. Daneben werden Basismaßnahmen bei akuten Kopfschmerzen - wie z.B. Rückzug, Reizabschirmung, Schaffen einer Schlafmöglichkeit - erläutert [14]. Als wichtiges Instrument sollte hier ein Kopfschmerztagebuch bzw. eines Aktivitätstagebuchs angeführt werden, um die Eigenwahrnehmung und Reflexion aktiv anzuregen [20]. Das gleiche Ziel wird mit der Bitte um Anfertigung einer grafischen/bildlichen Darstellung der erlebten Symptome verfolgt [20]. Ergänzend zum Gespräch kann auf internetbasierte Schulungsprogramme hingewiesen werden (z. B. Mütze hat den Kopfschmerz satt; z. B. auf Youtube zu sehen; [14]).

\section{Standardisierte Fragebögen - Lebensqualität, Kopfschmerz, Migräne}

Standardisierte Fragebögen sind ein bewährtes Instrument zur Primär- und Verlaufsbeurteilung der Krankheitslast. Für Kinder und Jugendliche stehen folgende zur Verfügung: Headache Impact Test (HIT, nicht validiert für die Altersgruppe, nach klinischer Erfahrung aber ab dem 14. Lebensjahr einsetzbar); Pediatric Migraine Disability Assessment Score (PedMIDAS); Pediatric Quality of Life Inventory (PedsQL); Fragebogen zur Lebensqualität von Kindern und Jugendlichen (KINDL) [14, 42].

\section{Psychologische Diagnostik, Beratung und Intervention}

Krankheits- und Stressbewältigung sowie Selbstwahrnehmung und Selbstwirksamkeit sind Kernaspekte der psychologischen Arbeit mit Kindern und Jugendlichen, die an Migräne leiden. Psychologische Interventionen zur Stressmodifikation, Verbesserung der Körperund Selbstwahrnehmung, Stärkung der Autonomie und Erarbeiten von Verarbeitungsstrategien gelten als wirksame Maßnahmen [14]. Je nach individueller
Situation zielt die Diagnostik auf: Identifikation von Auslösern oder „Unterhaltern" des Kopfschmerzes (z. B. Stressund Stresswahrnehmung, Überforderung, sekundärer Krankheitsgewinn, Schulvermeidungsverhalten), Objektivierung von Folgen der Schmerzerfahrung (z.B. Einbuße hinsichtlich Konzentrationsfähigkeit, Leistungsniveau), kognitive Leistungstestung (Erkennen von Überforderungssituationen in der Schule), Erkennen von psychischen Belastungen, emotionalen Schwierigkeiten und psychiatrischen Komorbiditäten (z.B. Hyperaktivität, Angststörungen, Depression, posttraumatische Belastungsstörung; [43, 44]). Die Autoren haben 2019 im Rahmen des Innovationsfondsprojektes „Modules on Migraine Aktivity - moma" des Gemeinsamen Bundesauschusses (G-BA) und der Ludwig-Maximilians-Universität München (LMU) zur Visualisierung der verschiedenen Faktoren ein Holzriesenrad entwickelt. Durch das spielerische Sortieren von Gewichten in den Gondeln wird die Bedeutung einzelner Faktoren begreifbar für die jungen Patienten. Dieses Konzept wird bisher mit bemerkenswerter Akzeptanz bei Kindern und Eltern im Rahmen des o.g. Projekts eingesetzt [45].

\section{Physiotherapeutische Diagnostik, Beratung und Intervention}

Der Schwerpunkt der physiotherapeutischen Diagnostik liegt auf der Beurteilung der Schulter-Nacken-Muskulatur mit Fokus auf Druckdolenzen, Verspannungen und myofaszialen Triggerpunkten $(\mathrm{m} T r \mathrm{P})$ sowie der Körperhaltung. Die Beratung umfasst die Aufklärung über den Stellenwert körperlicher Aktivität sowie Anleitungen zu Eigenübungen (z. B. Haltungs- und Dehnübungen). Physiotherapeutische Behandlung der SchulterNacken-Muskulatur kann in diesen Fällen von Wärmeanwendungen, über passives Dehnen, manuelle Techniken bis hin zur spezifischen Triggerpunkttherapie reichen [9].

\section{Pharmakotherapie - akute Migräneattacken}

Für akute Migräneattacken sollte jeder Patient über ein zuverlässig analgetisches Medikament verfügen, dass er frühzeitig und in ausreichend hoher Dosierung einnimmt. Bei unzureichender Wirksamkeit der Analgetika (30-60\% der Patienten) stellen die migränespezifischen Triptane eine Alternative dar, ggf. auch in Kombination mit einem langwirksamen nichtsteroidalen Antiphlogistikum (NSAID, z. B. Naproxen). Der Einsatz von ausgewählten Antiemetika kann im Einzelfall bei starker Übelkeit sinnvoll sein (z. B. Ondansetron $[14,15,46]$ ).

\section{Pharmakoprophylaxe}

Die Indikation für eine Pharmakoprophylaxe - abgesehen von der Einnahme von Magnesium - wird im Kindesund Jugendalter aufgrund der zumeist schlechten Nutzen-Risiko-Abwägung und der fehlenden Zulassung sowie der limitierten Evidenz selten gestellt. Sie kommt in der Regel erst dann für Einzelfälle in Betracht, wenn die psychologischen und nichtmedikamentösen Maßnahmen ausgeschöpft sind [sic!] und trotzdem folgende Situationen vorliegen: hohe Attackenfrequenz (Migräne $>1-2 \times /$ Woche oder $>3-4 \times$ /Monat) oder chronischer täglicher Kopfschmerz; relevante Einschränkung in Bezug auf Schulpräsenz, Alltagsfunktionalität, Aktivitäten (z. B. Migräne PedMIDAS $\geq 30$ ) und Lebensqualität; unzureichende Wirksamkeit und Verträglichkeit oder regelmäßige Überdosierung der Akuttherapeutika; Kontraindikation für die Akuttherapeutika; prolongierte Migräne ( $>48 \mathrm{~h})$, hemiplegische Migräne und Migräne mit Hirnstammaura (früher: Basilarismigräne) sowie ausgeprägte andere Auren (als nicht evidenzbasierte Indikationen; $[15,16])$.

Für die Anwendung der neuen Wirkgruppe der Calcitonin-gene-relatedpeptide-(CGRP-)Antikörper (bzw. Rezeptorantikörper) fehlen bislang pädiatrische Daten [47]. Hier ist in Zukunft kritisch abzuwarten, ob diese Substanzgruppe bei dem prophylaktischen Primat von Physiotherapie, Psychologie 
und Selbstwirksamkeit bei Kindern und Jugendlichen tatsächlich einen Zusatznutzen gegenüber der konsequenten Ausschöpfung nichtpharmakologischer Maßnahmen zeigt.

\section{Komplementärmedizinische Therapieansätze}

Die Nachfrage nach komplementärmedizinischen Therapiemöglichkeiten ist unverändert hoch. Die Familien sollten dahin gehend beraten werden, dass hinsichtlich der Spezifika des Kindes- und Jugendalters keine ausreichend belastbaren evidenzbasierten Daten vorliegen $[46,48]$.

\section{Pathophysiologie der Migräne - aktuelle Konzepte}

Die Migräne ist ein komplexes Krankheitsbild. Aus einer ursprünglich rein vaskulären Hypothese, die sich vor allem auf die Vasodilatation der meningealen Gefäße stützte, hat sich im Laufe der Zeit eine neuronale Hypothese mit zentraler und peripherer Komponente entwickelt [49-52]. Insgesamt wird die Migräne heute, wie oben bereits angerissen, als ein sich phasenweise verändernder Funktionszustand des Gehirns verstanden (,migraine is a brain state“; [36]).

Das Gehirn eines Menschen mit Migräne zeigt gegenüber Menschen ohne Migräne eine veränderte Schwelle der (Schmerz-)Reizwahrnehmung sowohl in einer akuten Migräneepisode, aber auch im Intervall [53]. Diese funktionellen Veränderungen in einem Hypothalamus-Thalamus-Hirnstamm-

Netzwerk oszillieren biologisch und chronobiologisch sowohl bezogen auf die jeweilige, einzelne Schmerzattacke als auch bezogen auf längere Wochen bis Monate andauernde Zyklen. Den Rhythmus für diese „Phasen unterschiedlicher Suszeptibilität" geben sogenannte Migränegeneratoren (im Hypothalamus und Hirnstamm, sog. Taktgeber). Je niedriger diese Reizschwelle, umso eher können individuelle Triggerfaktoren zur Auslösung einer Migräneattacke führen, die - wiederum zu betonen - so viel mehr ist als „nur“ ein Kopfschmerz. In der multifaktoriellen Genese spielen genetische (in der Regel nicht monogenetische) Faktoren eine auch in der klinischen Gewichtung wichtige Rolle, wie die häufig positive Familienanamnese veranschaulicht [54].

Stress als Faktor im Rahmen einer Migräneerkrankung wird vor diesem Hintergrund von zwei Seiten beleuchtet. Zum einen kann körperlicher/ psychischer Stress (oder sein plötzlicher Abfall) in Phasen einer niedrigen Reizschwelle die Migränekaskade in Gang setzen [53]. Auf der anderen Seite zeigen Untersuchungen bei Gymnasiasten, dass Jugendliche mit Migräne ein anderes Stresserleben zeigen, i.S. einer höheren Sensitivität $[9,43]$. Diese ließe sich auf Grundlage der bei Migräne in jeder Phase veränderten Reizwahrnehmung als Ergebnis der insgesamt und multimodal reduzierten Habituation einordnen. Luedtke et al. konnten nachweisen, dass Migränepatienten mit einer initial (und nur initial) erhöhten Muskelanspannung als Stressantwort auf eine akute psychische und physische Stresssituation reagieren [55]. Eine Beobachtung, die auch zum „Dazugehören“ peripherer Komponenten in der Komplexität der pathophysiologischen Vorgänge weitergedacht werden kann.

Für die periphere Komponente in der Migränepathophysiologie spielt die Konzeptualisierung als sogenannter trigeminozervikaler Komplex (TCC) eine wichtige Rolle. Hierunter wird eine peripher-zentrale Verknüpfung verstanden, welche im Wesentlichen die Konvergenz von zervikalem nozizeptiven Input (C1C3) in den kaudalen Nuclei des Nervus trigeminus im Hirnstamm beschreibt $[50,56]$. Auf Basis des TCC gelingt eine Verbindung von Migräne zu den praktisch so evidenten Nackenschmerzen. Die Assoziation von Schmerzsymptomen der Schulter-Nacken-Region und Migräne konnte im Rahmen epidemiologischer Studien nachgewiesen werden. $7 \%$ der Menschen mit Migräne geben an, in der Zeit der Prodromalphase, also bis zu $24 \mathrm{~h}$ vor Beginn der Kopfschmerzen, unter Nackenschmerzen zu leiden. $25 \%$ der Menschen mit Migräne leiden während der Kopfschmerzen zusätzlich an Nackenschmerzen $[57,58]$. Verspannungen im Nacken-Schulter-Bereich bei
Kindern und Jugendlichen, die an Kopfschmerzen - insbesondere Migräne leiden, sind häufig [59-61].

Betroffen sind hiervon insbesondere die C1-C3-versorgten $\mathrm{Mm}$. trapezii, $\mathrm{Mm}$. splenii/semispinales capites und Mm. sternocleidomastoidei - womit der Brückenschlag zum TCC gelingt. Oft sind in diesen Muskeln sog. myofasziale Triggerpunkte ( $\mathrm{mTrP}$ ) nachweisbar [62]. MTrP werden als umschriebene, tastbar verhärtete Strukturen in einem straffen Muskelstrang (Taut Band) eines Skelettmuskels definiert, die bei manueller Palpation eine lokale Muskelzuckung (Twitch Response), Druckschmerzhaftigkeit (Jump Sign) und/ oder ausstrahlende Missempfindungen bis hin zum dem Patienten bekannten Kopfschmerz (Referred Sensation, Referred Pain) zeigen [63]. Es ist belegt, dass durch manuelle Palpation myofaszialer Triggerpunkte bei Kindern und Jugendlichen Migränekopfschmerzen ausgelöst werden können [32].

Als Goldstandard zur Diagnose von $\mathrm{mTrP}$ gilt bislang die manuelle Palpation, deren Schwäche eindeutig in der nicht objektivierbaren Reproduzierbarkeit liegt [63]. Studien zu objektiven bildgebenden Verfahren - wie Sonographie oder Elastographie und Infrarotthermographie - blieben bisher kontrovers, ohne konklusive Ergebnisse [64]. Mittels hochauflösender 3-Tesla-Magnetresonanztomographie (MRT) gelang es bei jungen Erwachsenen zwar in der T2-gewichteten Darstellung hyperintense Veränderungen in als mTrP-palpierten Bereichen des M. trapezius darzustellen [65], eine verblindete Beurteilung zeigte aber mangelnde Reliabilität (Publikation under review). Mittels neuer bildgebender quantitativer Verfahren (T2-Kartierung) gelang 2018 erstmals eine objektivierbare und reproduzierbare Darstellung von $m \operatorname{TrP}$ [66]. Lassen sich diese Ergebnisse in weiteren Untersuchungen bestätigen, werden für wissenschaftliche Studien zukünftig objektive Messverfahren zur Verfügung stehen, um $\mathrm{mTrP}$ in ihrer Beziehung zur Migräne und mit ihren Veränderungen durch natürlichen Verlauf und Therapien zu dokumentieren. 
Tab. 1 Übersicht der zum aktuellen Zeitpunkt verfügbaren und erprobten nichtinvasiven zentralen und peripheren Methoden der Neuromodulation bei Migräne [17, 51, 69, 71, 72]

\begin{tabular}{|c|c|c|c|c|c|c|}
\hline \multirow[t]{2}{*}{ Methode } & \multirow[t]{2}{*}{$\begin{array}{l}\text { Technik } \\
\text { ES/MI }\end{array}$} & \multirow[t]{2}{*}{ Stimulationsort } & \multirow{2}{*}{$\begin{array}{l}\text { Einsatz in } \\
\text { Studien } \\
\text { zur Akut- } \\
\text { therapie } \\
\text { Ja+/ } \\
\text { Nein- }\end{array}$} & \multirow{2}{*}{$\begin{array}{l}\text { Einsatz in } \\
\text { Studien } \\
\text { zur Pro- } \\
\text { phylaxe } \\
\text { Ja+/ } \\
\text { Nein- }\end{array}$} & \multicolumn{2}{|c|}{$\begin{array}{l}\text { Zulassungsstatus für ver- } \\
\text { fügbare Medizinprodukte } \\
\text { für die Migränebehand- } \\
\text { lung }\end{array}$} \\
\hline & & & & & $\begin{array}{l}\text { Europa (CE) } \\
\text { Ja/Nein; } \\
\text { Indikation }\end{array}$ & $\begin{array}{l}\text { USA (FDA) } \\
\text { Ja/Nein; } \\
\text { Indikation }\end{array}$ \\
\hline (r)TMS & MI & $\begin{array}{l}\text { Variabel in Studien! Über dem okzipitalen Kortex (Akuttherapie, } \\
\text { Migräne mit visueller Aura, TMS Einzelimpulse; Prophylaxe, Migrä- } \\
\text { ne } \pm \text { Aura, TMS Einzelimpulse); über dem dorsolateralen präfron- } \\
\text { talen Kortex (Prophylaxe, chronische Migräne, rTMS); über dem } \\
\text { Handareal des linken Motorkortex (Prophylaxe, Migräne } \pm \text { Aura } \\
\text { bzw. chronische Migräne, rTMS) }\end{array}$ & + & + & $\begin{array}{l}\text { Ja; Akut- } \\
\text { therapie, } \\
\text { Prophylaxe }\end{array}$ & $\begin{array}{l}\text { Ja; Akut- } \\
\text { therapie, } \\
\text { Prophylaxe }\end{array}$ \\
\hline tDCS & ES & $\begin{array}{l}\text { Variabel in Studien! Über dem linken primären Motorkortex bzw. } \\
\text { dem dorsolateralen präfrontalen Kortex (Prophylaxe, medikamen- } \\
\text { tös refraktäre, chronische Migräne); über dem okzipitalen Kortex } \\
\text { (Prophylaxe) }\end{array}$ & - & + & $\begin{array}{l}\text { Schmerz } \\
\text { (keine spe- } \\
\text { zifischere } \\
\text { Angabe) }\end{array}$ & Nein \\
\hline $\begin{array}{l}\text { tONS }+ \\
\text { tSNS }\end{array}$ & ES & $\begin{array}{l}\text { Supraorbitale/supratrochleare Austrittspunkte des N. trigemi- } \\
\text { nus + N. occipitalis major bds. }\end{array}$ & + & - & $\begin{array}{l}\text { Ja; Akutthe- } \\
\text { rapie }\end{array}$ & Nein \\
\hline tSNS & ES & Supraorbitale/supratrochleare Austrittspunkte des N. trigeminus & + & + & $\begin{array}{l}\text { Ja; Akut- } \\
\text { therapie, } \\
\text { Prophylaxe }\end{array}$ & $\begin{array}{l}\text { Ja; Akut- } \\
\text { therapie, } \\
\text { Prophylaxe }\end{array}$ \\
\hline \multirow[t]{2}{*}{ tVNS } & \multirow[t]{2}{*}{ ES } & Zervikaler N. vagus: medial des M. sternocleidomastoideus & + & + & $\begin{array}{l}\text { Ja; Akut- } \\
\text { therapie, } \\
\text { Prophylaxe }\end{array}$ & $\begin{array}{l}\text { Ja; Akutthe- } \\
\text { rapie }\end{array}$ \\
\hline & & Aurikulärer N. vagus: äußerer Gehörgang & - & + & $\begin{array}{l}\text { Ja; Akut- } \\
\text { therapie, } \\
\text { Prophylaxe }\end{array}$ & Nein \\
\hline REN & ES & $\begin{array}{l}\text { Lateraler Oberarm, zwischen M. deltoideus und M. triceps; Ziel- } \\
\text { struktur: oberflächliche Hautnerven }\end{array}$ & + & - & Nein & $\begin{array}{l}\text { Ja; Akutthe- } \\
\text { rapie }\end{array}$ \\
\hline rPMS & $\mathrm{Ml}$ & M. trapezius (myofasziale Triggerpunktareale) & - & + & Nein & Nein \\
\hline
\end{tabular}

\section{Nichtpharmakologische innovative Therapieansätze}

Neben den Weiterentwicklungen der pharmakologischen Konzepte wird zunehmend der Bedarf an nichtpharmakologischen Therapieansätzen betont. Unter pathophysiologischen Gesichtspunkten werden diese Methoden als neuromodulatorische Therapien bezeichnet, da sie auf die Schmerzverarbeitungsprozesse einwirken. Prinzipiell sind periphere und zentrale neuromodulatorische Ansätze sowie deren akuter oder prophylaktischer Einsatz zu unterscheiden. Einen Überblick über die nichtinvasiven Methoden zur Behandlung der Migräne gibt - Tab. 1. Die spezifischen Wirkmechanismen und pathophysiologischen Vorgänge sind dabei nach wie vor Gegenstand intensiver Forschung einzelner Arbeitsgruppen [46]. Wie oben aufgeführt, führt die Migräne zu einer oszillierenden Veränderung zentraler Triggerschwellen, wobei periphere Eingänge, wie z. B. die nozizeptiven Fasern aus dem trigeminalen als auch zervikalen Bereich, eine Rolle spielen. Durch elektrische Reizung im Gebiet des N. supraorbitalis kann die Migränefrequenz bei episodischer Migräne signifikant reduziert werden [17]. Für die positive Wirkung der Stimulation zervikaler Afferenzen gibt es erste Hinweise (z. B. rPMS, s. unten; [17, 51]). Die Modulation dieser Afferenzen erfolgt über die Induktion elektrischer Ströme im Körpergewebe - entweder durch Elektro- oder magnetische Stimulation. Insbesondere Patienten, bei denen Kontraindikationen für medikamentöse Migränetherapeutika bestehen, die deren Nebenwirkungen nicht tolerieren oder grundsätzlich nicht auf die Pharmakotherapie ansprechen, wird so eine neue Alternative eröffnet [67]. Zur Zielgruppe für nichtinvasive und sichere i. S. von nebenwirkungsarmen Interventionen zählen nach Meinung der Autoren prioritär Kinder und Jugendliche [68].

Insgesamt ist die Evidenzlage nicht für alle Anwendungen ausreichend hoch. Eine Schwierigkeit liegt hierbei in der Etablierung einer methodisch adäquaten Sham-Kontrolle. Daneben liegen bislang keine kontrollierten Daten zum Vergleich der Verfahren untereinander, zur Kombination verschiedener Verfahren oder zum Einfluss von parallel eingenommener Medikation vor. Ebenso sind 
die Daten zum Vergleich der Wirksamkeit bei verschiedenen Patientengruppen (z.B. Alter, Geschlecht, episodische Migräne, chronische Migräne, Aura positiv/ negativ, Krankheitsdauer, Krankheitslast) und Daten zur Nachhaltigkeit der Effekte nicht ausreichend vorhanden. Des Weiteren fehlen Vergleiche unterschiedlicher Stimulationsprotokolle und Settings. Auch wenn auf dem Markt nun verschiedene Geräte zur selbstständigen Anwendung verfügbar sind, sollte die Auswahl auf individueller Basis - auch vor dem Hintergrund der entstehenden Kosten - gemeinsam getroffen und die Behandlungen sollten gut angeleitet und dokumentiert werden $[16,17,69]$.

Für keine der dargestellten Interventionen existieren bisher Daten zur Anwendung im Kindes- und Jugendalter. Aufgrund der guten Akzeptanz, Sicherheit und Wirksamkeit einer Behandlung mittels repetitiver peripherer Magnetstimulation im Bereich der SchulterNacken-Muskulatur bei jungen Erwachsenen mit hochfrequenter episodischer Migräne könnten entsprechende Untersuchungen bei ausgewählten Kindern und Jugendlichen (episodische Migräne + myofasziale Triggerpunkte Schulter-Nacken-Muskulatur) folgen [19, 20]. Gerade in dieser Altersgruppe wird häufig die Assoziation muskulärer Symptome zur Migräne beschrieben, die über den TCC konzeptualisiert werden kann. Durch die im Bereich der Schulter-Nacken-Muskulatur ansetzende Intervention eröffnet sich die Chance, von peripher her neuromodulierend auf zentrale Schmerzmechanismen einzuwirken und in der Folge eine Linderung von Migränesymptomen zu erreichen. Aufgrund der Verfügbarkeit von Magnetstimulationsgeräten, die für die ambulante, physiotherapeutische Anwendung konzipiert wurden, kann diese Art der Behandlung im Jahr 2020 auch außerhalb neurophysiologischer Forschungslabore in einem kindgerechten Setting evaluiert werden. Vorteile dieser Methode sind die Schmerzlosigkeit, das Fehlen von Elektroden, die angebracht werden müssen, und die Anwendung in Bekleidung [70]. In Analogie zur transkraniellen Magnetstimulation (TMS), deren Sicherheit im Kindesalter in ei- ner Metaanalyse belegt wurde, ist auch bei der peripheren Magnetstimulation (PMS) von einem guten Sicherheitsprofil auszugehen [68].

\section{Fazit}

Die Migräne ist ein häufiges und für den einzelnen Patienten extrem relevantes Krankheitsbild. Betroffenen Kindern und Jugendlichen sollte ein multimodales Therapiekonzept angeboten werden können, falls die Standardmaßnahmen nicht ausreichen, sich ein komplizierter Verlauf abzeichnet oder eine komplexe Gesamtsituation besteht. Aufgrund der nicht zufriedenstellenden Nutzen-Risiko-Abwägung für die meisten Pharmakotherapien rücken zunehmend nichtpharmakologische Behandlungsansätze in den Vordergrund. Durch Neurostimulation werden hier die zentralen, aber auch peripheren Mechanismen der Schmerzverarbeitung für den Patienten positiv beeinflusst. Diese innovativen, neuromodulatorischen Therapien sollten - zunächst im Rahmen von Studien - auch Kindern und Jugendlichen zugänglich gemacht werden. Die SchulterNacken-Muskulatur als ein klinisch gut zugängliches „Markerorgan der Migräne"lässt Rückschlüsse auf die individuelle Bedeutung der (1) lokalen, myofaszialen Komponente, (2) der zentrofugalen und zentripetalen Komponente des trigeminozervikalen Schmerzkomplexes und (3) die individuelle Körperreaktion auf Stress zu. Pathophysiologisch-theoretisch lassen sich die positiven Effekte der repetitiven peripheren Magnetstimulation rPMS sowohl (1) über das Konzept des trigeminozervikalen Komplexes als auch (2) über das Konzept der peripheren Sensitivierung des kaudalen Anteiles des Kerngebietes des N. trigeminus als auch (3) über die myofaszialen Triggerpunkte begründen.

\section{Korrespondenzadresse}

Dr. med. Michaela Veronika Bonfert

Abteilung für Pädiatrische Neurologie, Entwicklungsneurologie, Sozialpädiatrie und LMU Zentrum für Entwicklung und komplex chronisch kranke Kinder - iSPZ Hauner, Dr. von Haunersches Kinderspital, LMU Klinikum Lindwurmstr. 4, 80337 München, Deutschland michaela.bonfert@med.Imu.de

Funding. Open Access funding provided by Projekt DEAL.

\section{Einhaltung ethischer Richtlinien}

Interessenkonflikt. M.V. Bonfert besitzt zum Zeitpunkt der Erstellung des Manuskripts ein Stipendium im Rahmen der Bayerischen Gleichstellungsförderung F. Heinen und M.N. Landgraf sind als kinderneurologische Konsortionalpartner und in diesem Rahmen Projektleitung im G-BAInnovationsfond Projektmoma („,modules on migraine acitivity ${ }^{\prime)}$ tätig. S.M. Krieg ist Berater für Brainlab AG (München, Deutschland), Nexstim Plc (Helsinki, Finnland), Spineart (Genf, Schweiz) und Zeiss Meditec (Oberkochen, Deutschland). N. Sollmann erhielt Honorare von Nexstim Plc (Helsinki, Finland). Im iSPZ Campus Hauner steht zum Zeitpunkt der Manuskripterstellung als vertraglich geregelte kostenfreie Leihgabe ein Gerät zur Magnetstimulation der Firma Zimmer MedizinSystem GmbH, Neu-Ulm, Deutschland (eMFieldPro) zur Verfügung. C. Börner, L. Gerstl, I. Hannibal, N. Mathonia, K. Huß, B. Rahmsdorf, C. Kainz, B. Klose, H. Koenig, G. Urban, P. Schandelmaier, T. Renner und L. Albers geben an, dass kein Interessenkonflikt besteht.

Für diesen Beitrag wurden von den Autoren keine Studien an Menschen oder Tieren durchgeführt. Für die aufgeführten Studien gelten die jeweils dort angegebenen ethischen Richtlinien.

Open Access. Dieser Artikel wird unter der Creative Commons Namensnennung 4.0 International Lizenz veröffentlicht, welche die Nutzung, Vervielfältigung, Bearbeitung, Verbreitung und Wiedergabe in jeglichem Medium und Format erlaubt, sofern Sie den/die ursprünglichen Autor(en) und die Quelle ordnungsgemäß nennen, einen Link zur Creative Commons Lizenz beifügen und angeben, ob Änderungen vorgenommen wurden.

Die in diesem Artikel enthaltenen Bilder und sonstiges Drittmaterial unterliegen ebenfalls der genannten Creative Commons Lizenz, sofern sich aus der Abbildungslegende nichts anderes ergibt. Sofern das betreffende Material nicht unter der genannten Creative Commons Lizenz steht und die betreffende Handlung nicht nach gesetzlichen Vorschriften erlaubt ist, ist für die oben aufgeführten Weiterverwendungen des Materials die Einwilligung des jeweiligen Rechteinhabers einzuholen.

Weitere Details zur Lizenz entnehmen Sie bitte der Lizenzinformation auf http://creativecommons.org/ licenses/by/4.0/deed.de. 


\section{Literatur}

1. Global Burden of Disease 2016 Headache Collaborators (2018) Global, regional, and national burden of migraine and tension-type headache 1990-2016: a systematic analysis for the Global Burden of Disease Study 2016. Lancet Neurol 17(11):954-976

2. Steiner TJ, Stovner LJ, Vos T, Jensen R, Katsarava Z (2018) Migraine is first cause of disability in under 50s: will health politicians now take notice? JHeadache Pain 19(1):17

3. Woldeamanuel YW, Cowan RP (2017) Migraine affects 1 in 10 people worldwide featuring recent rise: A systematic review and meta-analysis of community-based studies involving 6 million participants.J Neurol Sci 372:307-315

4. Stovner LJ, Andree C (2010) Prevalence of headache in Europe: a review for the Eurolight project. J Headache Pain 11(4):289-299

5. Ziegeler C, Brauns G, Jurgens TP, May A (2019) Shortcomings and missed potentials in the management of migraine patients - experiences from a specialized tertiary care center. J Headache Pain 20(1):86

6. Albers L, Straube A, Landgraf MN, Filippopulos F, Heinen F, von Kries R (2015) Migraine and tension type headache in adolescents at grammar school in Germany-Burden of disease and health care utilization. J Headache Pain 16:534

7. Albers L, von Kries R, Heinen F, Straube A (2015) Headache in school children: is the prevalence increasing? Curr Pain Headache Rep 19(3):4

8. Straube A, Andreou A (2019) Primary headaches during lifespan. J Headache Pain 20(1):35

9. Landgraf MN, Konig H, Hannibal I et al (2017) Migraine in children and adolescents-brain and muscle?: Another example of why children are not small adults. Nervenarzt 88(12):1402-1410

10. Miller S, Matharu MS (2014) Migraine is underdiagnosed and undertreated. Practitioner 258(1774):2-24

11. Straube A, Heinen F, Ebinger F, von Kries R (2013) Headache in school children: prevalence and risk factors. Dtsch Arztebl Int 110(48):811-818

12. Albers L, Straube A, Landgraf MN, Heinen F, von Kries R (2014) High diagnostic stability of confirmed migraine and confirmed tensiontype headache according to the ICHD-3 beta in adolescents.J Headache Pain 15:36

13. Milde-Busch A, Heinrich S, Thomas S et al (2010) Quality of life in adolescents with headache: results from a population-based survey. Cephalalgia 30(6):713-721

14. Bonfert MV, Landgraf MN, Mathonia NM et al (2019) Primäre Kopfschmerzen bei Kindern und Jugendlichen - Update 2019. Pädiatire Up2date 14(01):71-85

15. Bonfert M, Straube A, Schroeder AS, Reilich $P$, Ebinger $F$, Heinen $F$ (2013) Primary headache in children and adolescents: update on pharmacotherapy of migraine and tension-type headache. Neuropediatrics 44(1):3-19

16. Diener HC, Charles A, Goadsby PJ, Holle D (2015) New therapeutic approaches for the prevention and treatment of migraine. Lancet Neurol 14(10):1010-1022

17. Hoffmann J, May A (2019) Neuromodulation for the treatment of primary headache syndromes. ExpertRev Neurother 19(3):261-268

18. Rapoport AM, McAllister P (2020) The headache pipeline: excitement and uncertainty. Headache 60(1):190-199
19. Renner T, Sollmann N, Trepte-Freisleder F et al (2019) Repetitive peripheral magnetic stimulation (rPMS) in subjects with migraine-setup presentation and effects on skeletal musculature. Front Neurol 10:738

20. Sollmann N, Trepte-Freisleder F, Albers L et al (2016) Magnetic stimulation of the upper trapezius muscles in patients with migraine-A pilot study. Eur JPaediatr Neurol 20(6):888-897

21. Albers L, Kries RV, Straube A et al (2019) Age- and sex-specific first health care use for migraine in 2016 in children and adolescents from prospectively collected health insurance data in Germany. Cephalalgia. https://doi.org/10.1177/ 0333102419844543

22. Philipp J, Zeiler M, Wober C et al (2019) Prevalence and burden of headache in children and adolescents in Austria- a nationwide study in a representative sample of pupils aged 10-18 years. JHeadache Pain 20(1):101

23. Balottin U, Borgatti R, Zambrino CA, Lanzi G (1997) Clinical characteristics and long-term outcome of migraine with aura in children and adolescents. Dev Med Child Neurol 39(1):26-30

24. Sillanpaa M, Aro H (2000) Headache in teenagers: comorbidity and prognosis. Funct Neurol 15(Suppl 3):116-121

25. Sillanpaa M, Saarinen MM (2018) Long term outcome of childhood onset headache: A prospective community study. Cephalalgia 38(6):1159-1166

26. Buse DC, Greisman JD, Baigi K, Lipton RB (2019) Migraine progression: a systematic review. Headache 59(3):306-338

27. Lipton RB, Fanning KM, Buse DC et al (2019) Migraine progression in subgroups of migraine based on comorbidities: Results of the CaMEO Study. Neurology 93(24):e2224-e2236

28. Katsarava Z, Mania M, Lampl C, Herberhold J, Steiner TJ (2018) Poor medical care for people with migraine in Europe-Evidence from the Euroligh study. J Headache Pain 19(1):10

29. Martelletti $P$, Schwedt TJ, Lanteri-Minet $M$ et al (2018) My Migraine Voice survey: a global study of disease burden among individuals with migraine for whom preventive treatments have failed. JHeadache Pain 19(1):115

30. Buse DC, Fanning KM, Reed ML et al (2019) Life with migraine: effects on relationships, career, and finances from the chronic migraine epidemiology and outcomes (caMEO) study. Headache 59(8):1286-1299

31. Leonardi M, Raggi A (2019) A narrative review on the burden of migraine: when the burden is the impact on people's life. J Headache Pain 20(1):41

32. Landgraf MN, Biebl JT, Langhagen T et al (2018) Children with migraine: Provocation of headache via pressure to myofascial trigger points in the trapezius muscle?-A prospective controlled observational study. Eur J Pain 22(2):385-392

33. Saylor D, Steiner TJ (2018) The global burden of headache. Semin Neurol 38(2):182-190

34. Olesen J, Gustavsson A, Svensson Met al (2012) The economic cost of brain disorders in Europe. Eur J Neurol 19(1):155-162

35. Bonfert M, Landgraf M, Ebinger F, Heinen F (2016) Kopfschmerzen bei Kindern und Jugendlichen. In Gaul C, Diener HC (Hrsg) Kopfschmerzen. Thieme, Stuttgart

36. Charles A (2018) The pathophysiology of migraine: implications for clinical management. Lancet Neurol 17(2):174-182

37. Headache Classification Committee of the International Headache Society (2018) The Internationa
Classification of Headache Disorders, 3rd edition. Cephalalgia 38(1):1-211

38. Roser T, Bonfert M, Ebinger F, Blankenburg M, ErtlWagner B, Heinen F (2013) Primary versus secondary headache in children: a frequent diagnostic challenge in clinical routine. Neuropediatrics 44(1):34-39

39. Bonfert M, Landgraf M, Giese R, Klose B, Gerstl L, Heinen F (2014) Migraine in adolescence: a modular concept. Nervenheilkunde 2014(3):6

40. Albers L, Milde-Busch A, Bayer O et al (2013) Prevention of headache in adolescents: populationattributable risk fraction for risk factors amenable to intervention. Neuropediatrics 44(1):40-45

41. Milde-Busch A, Blaschek A, Borggrafe I, Heinen F, Straube A, von Kries R (2010) Associations of diet and lifestyle with headache in high-school students: results from a cross-sectional study. Headache 50(7):1104-1114

42. Office of Quality of Life Measures (2020) Fragebögen. https://www.kindl.org/deutsch/frageb\%C3 \%B6gen/.Zugegriffen:24. Mai 2020

43. Milde-Busch A, Blaschek A, Heinen F et al (2011) Associations between stress and migraine and tension-type headache: results from a schoolbased study in adolescents from grammar schools in Germany. Cephalalgia 31(7):774-785

44. Milde-Busch A, Boneberger A, Heinrich $S$ et al (2010) Higher prevalence of psychopathological symptoms in adolescents with headache. A population-based cross-sectional study. Headache 50(5):738-748

45. integriertes Sozialpädiatrisches Zentrum (iSPZ Hauner) (2020) moma modules on migraine activity. http://www.klinikum.uni-muenchen.de/ Integriertes-Sozialpaediatrisches-Zentrum-imDr-von-Haunerschen-Kinderspital/de/projekte/ moma/index.html. Zugegriffen: 24. Mai 2020

46. Diener HC, Gaul C, Kropp P (2018) Therapie der Migräneattacke und Prophylaxe der Migräne, S1Leitlinie. Nervenheilkunde 37:689-715

47. Tepper SJ (2018) Anti-calcitonin gene-related peptide (CGRP) therapies: Update on a previous review after the American Headache Society 60th Scientific Meeting, San Francisco, June 2018. Headache 58(Suppl 3):276-290

48. Schetzek S, Heinen F, Kruse S et al (2013) Headache in children: update on complementary treatments. Neuropediatrics 44(1):25-33

49. Burstein R, Noseda R, Borsook D (2015) Migraine: multiple processes, complex pathophysiology. JNeurosci 35(17):6619-6629

50. Akerman S, Romero-Reyes M, Holland PR (2017) Current and novel insights into the neurophysiology of migraine and its implicationsfor therapeutics. Pharmacol Ther 172:151-170

51. Rapoport AM, Edvinsson L (2019) Some aspects on the pathophysiology of migraine and a review of device therapies for migraine and cluster headache. Neurol Sci 40(Suppl 1):75-80

52. Puledda F, Messina R, Goadsby PJ (2017) An update on migraine: current understanding and future directions. J Neurol 264(9):2031-2039

53. Nahman-Averbuch $\mathrm{H}$, Leon $\mathrm{E}$, Hunter $\mathrm{BM}$ et al (2019) Increased pain sensitivity but normal pain modulation in adolescents with migraine. Pain 160(5):1019-1028

54. Ziegeler C, May A (2019) Pathophysiologie de Migräne. Aktuelles Verständnis und Ausblick. Nervenheilkunde 38(10):722-727

55. Luedtke K, Mehnert J, May A (2018) Altered muscle activity during rest and during mental or physical activity is not a trait symptom of migraine-A neck muscle EMG study. J Headache Pain 19(1):26 


\section{Leitthema}

56. Bartsch T, Goadsby PJ (2003) The trigeminocervical complex and migraine: current concepts and synthesis. Curr Pain Headache Rep 7(5):371-376

57. Lampl C, Rudolph M, Deligianni Cl, Mitsikostas DD (2015) Neckpain in episodicmigraine:premonitory symptom or part of the attack? J Headache Pain 16:566

58. Castien R, De Hertog W (2019) A neuroscience perspective of physical treatment of headache and neck pain. Front Neurol 10:276

59. Blaschek A, Decke S, Albers L et al (2014) Selfreported neck pain is associated with migraine but not with tension-type headache in adolescents. Cephalalgia 34(11):895-903

60. Landgraf MN, von Kries R, Heinen F, Langhagen T, Straube A, Albers L (2016) Self-reported neck and shoulder pain in adolescents is associated with episodic and chronic migraine. Cephalalgia 36(8):807-811

61. Blaschek A, Milde-Busch A, Straube A et al (2012) Self-reported muscle pain in adolescents with migraine and tension-type headache. Cephalalgia 32(3):241-249

62. Do TP, Heldarskard GF, Kolding LT, Hvedstrup J, Schytz HW (2018) Myofascial trigger points in migraine and tension-type headache. J Headache Pain 19(1):84

63. Fernandez-de-Las-Penas C, Dommerholt J (2018) International consensus on diagnostic criteria and clinical considerations of myofascial trigger points: a Delphi study. Pain Med 19(1):142-150

64. Takla MK, RazekNM, Kattabei O, El-Lythy MA (2016) A comparison between different modes of realtime sonoelastography in visualizing myofascial trigger points in low back muscles. J Man Manip Ther 24(5):253-263

65. Landgraf MN, Ertl-Wagner B, Koerte IK et al (2015) Alterations in the trapezius muscle in young patients with migraine - a pilot case series with MRI. Eur J Paediatr Neurol 19(3):372-376

66. Sollmann N, Mathonia N, Weidlich D et al (2019) Quantitative magnetic resonance imaging of the upper trapezius muscles-Assessment of myofascial trigger points in patients with migraine. JHeadache Pain 20(1):8

67. Puledda F, Goadsby PJ (2017) An update on nonpharmacological neuromodulation for the acute and preventive treatment of migraine. Headache 57(4):685-691

68. Brighina F, Raieli V, Messina LM et al (2019) Noninvasive brain stimulation in pediatric migraine: a perspective from evidence in adult migraine. Front Neurol 10:364

69. Jurgens TP, Rimmele F (2019) Neuromodulation in primary headache in the year 2019: is it still up-to-date? New data on invasive and noninvasive neuromodulation in migraine and cluster headaches. Schmerz 33(4):347-367

70. Beaulieu LD, Schneider C (2015) Repetitive peripheral magnetic stimulation to reduce pain or improve sensorimotor impairments: A literature review on parameters of application and afferents recruitment. Clin Neurophysiol 45(3):223-237

71. Reuter U, McClure C, Liebler E, Pozo-Rosich P (2019) Non-invasive neuromodulation for migraine and cluster headache: a systematic review of clinical trials. JNeurol Neurosurg Psychiatry 90(7):796-804

72. Lipton RB, Goadsby PJ (2018) Comment: Noninvasive neurostimulation for migraine should be part of the general neurologist's therapeutic armamentarium. Neurology 91(4):167 